\title{
A VECTOR FIELD COLOR RENDITION MODEL FOR CHARACTERIZING COLOR SHIFTS AND METAMERIC MISMATCH
}

\author{
Aurelien David ${ }^{1}$, Tony Esposito ${ }^{2}$, Kevin Houser ${ }^{3}$, Michael Royer ${ }^{4}$, Kevin A.G. Smet ${ }^{5}$, Lorne Whitehead ${ }^{6}$ \\ ${ }^{1}$ Soraa, ${ }^{2}$ Esposito Research, ${ }^{3}$ Pennsylvania State University, ${ }^{4}$ Pacific Northwest National Laboratory, ${ }^{5}$ ESAT / Light \\ \& Lighting Laboratory, KU Leuven, ${ }^{6}$ Department of Physics and Astronomy, University of British Columbia
}

\begin{abstract}
This work distinguishes between two very different components of light-source-induced color shift—base color shift and metameric color shift-in order to provide a more complete understanding of color rendition. Working within the existing framework of IES TM-30-18 and CIE 224, we show that base color shift is a smoothly-varying function of location in color space, and it is strongly dependent on the light source SPD. We demonstrate that this variation can be represented well by a vector field model based on a second order polynomial vector function, the parameters of which are adjusted to provide a least squares fit to the calculated color shifts of the standard set of color samples. Once the base color shift model for a given source is determined, the residual metameric color shifts can be calculated, and the distribution of those random shifts can be statistically summarized. Based on that information, a proposed Metameric Uncertainty Index $\left(R_{\mathrm{t}}\right)$, is introduced to provide new information about a light source SPD.
\end{abstract}

\section{INTRODUCTION}

In this work, we build on the common color rendition framework established in IES TM-30 [IES 2015, 2018] and CIE 224:2017 [CIE 2017], which concern the shifts of objects' color appearance (here called color shifts) that occur when the illumination is changed from a reference to a test source. The framework specifies a set of spectral reflectance factors (here called color samples) for color shift evaluation by means of a perception-based color appearance model and a CCT-dependent reference illuminant. It also includes a set of useful color rendition measures.

That framework is now in widespread successful use worldwide; this paper does not propose any change to it. Rather, two additional useful color rendition measures are presented, which may also be helpful in developing future color rendition metrics for better predicting overall human experience. Both are based on an important observation from previous studies of large numbers of color samples representing real objects: For any selected light source, in any given region in color space, there is an average color shift (here called the base color shift), and a distribution of color shifts around that average (here called the metameric color shift).

In this work, we model base color shift throughout color space as a second order polynomial vector function, with the coefficients adjusted to yield the least squares best fit to the color shift data. Next, the average errors for the least squares fit are used to statistically characterize the metameric shifts of the color samples. This allows for the definition of a new useful average measure of color rendition, the Metameric Uncertainty Index $\left(R_{\mathrm{t}}\right)$.

\section{BASE COLOR SHIFT VERSUS METAMERIC COLOR SHIFT}

Figure 1 represents color coordinates for three different groups of color samples under a test SPD (dots) and reference illuminant SPD (crosses). Under the reference illuminant SPD, the samples in each group share a single color coordinate $\left(J^{\prime}, a^{\prime}, b^{\prime}\right)$ in the CAM02-UCS color space and hence plot to the same, single coordinate in the 2-D $\left(a^{\prime}, b^{\prime}\right)$ graph. The cloud-like scatter of points for the color samples under the test SPD shows how they shift in different directions. The points in each cloud can be described as a combination of a base color shift toward the center of the pattern and an additional metameric color shift, from that point to a given color sample, which arises from the lightsource-induced mismatch of metamerism. As will be illustrated in this work, the base shift varies smoothly throughout color space. In contrast, the metameric color shift is dependent on the specific color sample. Thus, metameric color shift is a source of uncertainty when color rendition measures, (based on standardized color samples), are used to predict the color shifts of real objects. As will be shown, some light sources induce relatively higher levels of this uncertainty, independent of other measures of color rendition, such as color fidelity. This also means that such light sources are more likely to lead to color mismatches where matches were intended. 


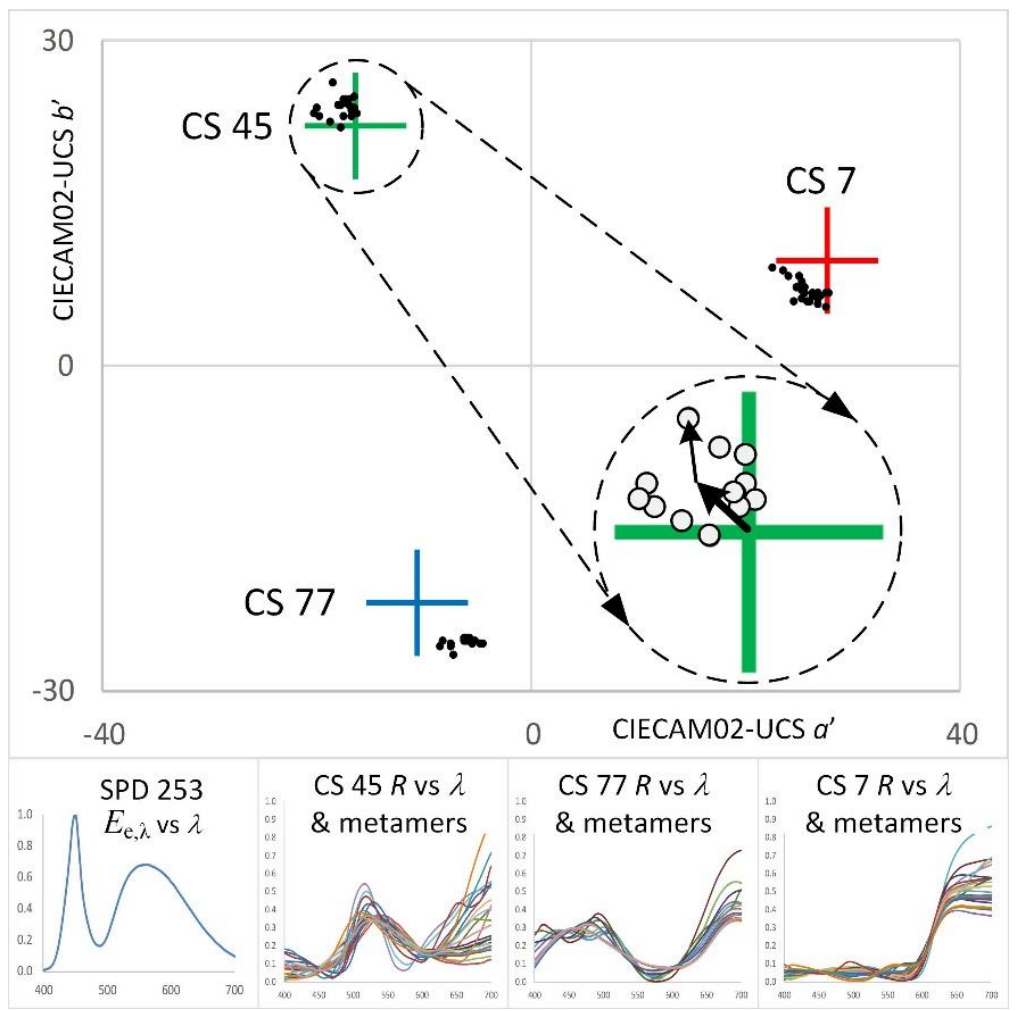

Figure 1. Illustration of light-source-induced color shift and its two components: base color shift and metameric color shift. Top: Three crosses represent three sets of SRFs under a TM-30/CIE 224 reference SPD, the scattered black dots represent the same SRFs under the test SPD. The enlargement in the lower right quadrant shows the base color shift as the bolder arrow and the metameric color shift for an example metameric SRF as the lighter arrow. Bottom: Relative SPD of the test light source and the SRFs of the three groups, with each group being metameric SRFs for the noted IES TM-30/CIE 224 color sample.

\section{VECTOR FIELD MODEL FOR COLOR SHIFTS}

We first checked to see if a simple first-order (linear) vector field model can reliably fit the actual color shifts with the desired accuracy, and it was found to be insufficient. However, the pattern of base color shifts is a smoothlyvarying continuous function of location in color space (presumably due to the smoothly-varying wavelength dependence of the cone fundamentals and reference illuminant SPD), and likely for this reason, only a slightly more complex function was needed. We used a general $2^{\text {nd }}$ order polynomial function of two variables, which has twelve free parameters as shown in Equations 1 and 2. $\Delta a_{m}^{\prime}$ is the modelled shift of coordinate $a^{\prime}$ for the test SPD versus the reference illuminant $\mathrm{SPD}$, and $\Delta b_{m}^{\prime}$ is the corresponding shift for coordinate $b^{\prime}$.

$$
\begin{aligned}
& \Delta a_{m}^{\prime}=c_{a 0}+c_{a 1} a^{\prime}+c_{a 2} b^{\prime}+c_{a 3} a^{\prime 2}+c_{a 4} a^{\prime} b^{\prime}+c_{a 5} b^{2} \\
& \Delta b_{m}^{\prime}=c_{b 0}+c_{b 1} a^{\prime}+c_{b 2} b^{\prime}+c_{b 3} a^{\prime 2}+c_{b 4} a^{\prime} b^{\prime}+c_{b 5} b^{\prime 2}
\end{aligned}
$$

Thus, for any selected $\left(a^{\prime}, b^{\prime}\right)$ coordinates, the vector field model predicts a base color shift. For a given color sample labeled by index value $i, \Delta a_{m i}^{\prime}$ and $\Delta b_{m i}^{\prime}$ depend on the 12 parameters that characterize the model. To reiterate, the goal is to determine values for the twelve coefficients that make $\Delta a_{m i}^{\prime}$ and $\Delta b_{m i}^{\prime}$ as close as possible to the actual calculated values, $\Delta a_{i}^{\prime}$ and $\Delta b^{\prime}$, for a given SPD. In general, the match cannot be perfect because a vector field model is only intended to predict the base color shift, not the metameric color shift that introduces random errors.

In practice, determining the coefficients in Equations 1 and 2 amounts to minimizing the sums of the squares of the errors, as defined in Equations 3 and 4. 


$$
\begin{aligned}
& E_{a}=\sum_{i=1}^{n}\left(a_{m i}^{\prime}-a_{i}^{\prime}\right)^{2} \\
& E_{b}=\sum_{i=1}^{n}\left(b_{m i}^{\prime}-b_{i}^{\prime}\right)^{2}
\end{aligned}
$$

Figure 2 shows examples of the vector field model determined both via equations 1 through 4 using the 99 color samples of IES TM-30 and CIE 224, and by an alternate approach using approximately 5000 color samples that are known to be highly uniform in color and wavelength space. The agreement shows that it is acceptable to use the existing IES TM-30 and CIE 224 color samples for this purpose; no new color samples are needed.

(a)
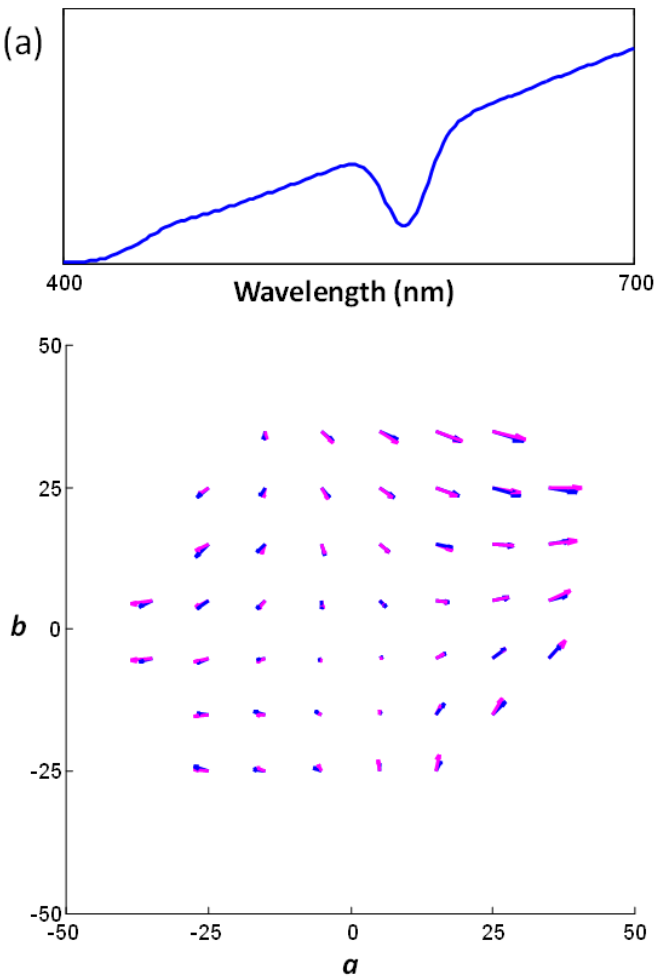

(b)
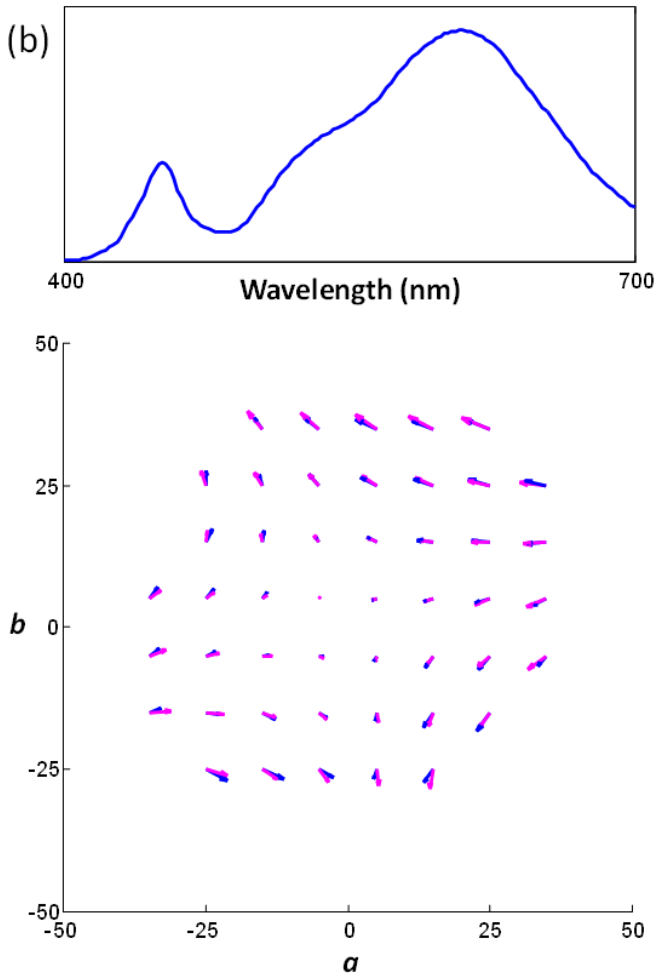

Figure 2. SPD and corresponding base color shifts in the $a^{\prime}-b^{\prime}$ plane, using 5,000 color samples (blue vectors) and using the 99 color samples of IES TM-30 and CIE 224). The example SPDs are (a) a neodymium lamp, (b) a phosphor-converted LED.

\section{METAMERIC UNCERTAINTY INDEX $\left(R_{\mathrm{t}}\right)$}

Having identified a recommended method for modelling base color shift, the second objective of this work was to establish a method for characterizing metameric color shift. The key information needed for this resides in the average errors that were minimized in adjusting the coefficients in the vector field model so as to best represent base color shift throughout color space. For any light source of interest, this yields a value for the Metameric Uncertainty Index, which provides supplemental information to the other color rendition measures of IES TM-30 and CIE 224. It is especially useful in situations where metameric mismatch might interfere with the color quality of illumination, such as in the textile industry or in retail display.

\section{CONCLUSION}

Because the vector field model specifically isolates base color shift, the remaining metameric color shift can also be determined. The Metameric Uncertainty Index $\left(R_{\mathrm{t}}\right)$ quantifies the uncertainty associated with measures of color rendition when the test light source SPD illuminates colored objects having unspecified spectral reflectance factors. It assesses the degree generalizability of color rendition calculations, and provides an estimate of the likelihood of metameric mismatch that might be induced by a given light source. The latter makes $R_{\mathrm{t}}$ a useful tool for any lighting applications where prevention of metameric mismatch is important. In general, the Metameric Uncertainty Index provides new information that complements other previously-established measures of color rendition. Both the vector field model and $R_{\mathrm{t}}$ are compatible with the IES TM-30-18/CIE 224:2017 framework. 